\title{
Concentração Plasmática de Ropivacaína Durante Anestesia Peridural Lombar em Crianças *
}

\section{Ropivacaine and Bupivacaine Plasma Concentration During Lumbar Epidural Anesthesia in Children}

Verônica Vieira da Costa ${ }^{1}$, Denise Pereira de Oliveira Souza ${ }^{1}$, Marcelo Buzzi ${ }^{2}$, Márcio Corrêa de Mello ${ }^{3}$, Renato Ângelo Saraiva TSA ${ }^{4}$

\begin{abstract}
RESUMO
Costa VV, Souza DPO, Buzzi M, Mello MC, Saraiva RA - Concentração Plasmática de Ropivacaína Durante Anestesia Peridural Lombar em Crianças
\end{abstract}

Justificativa e Objetivos - A ropivacaína é o mais novo anestésico local de uso na prática clínica. Sua estrutura é semelhante a forma levógira da bupivacaína, tendo portanto baixa toxicidade. Os valores das concentrações plasmáticas que podem ser atingidos em crianças, com o uso desta droga e também da bupivacaína (mesmo a forma racêmica) administradas por via peridural lombar, são ainda pouco conhecidos. O objetivo desse estudo foi avaliar as concentrações sangüíneas de ropivacaína e bupivacaína por via peridural lombar em crianças, em bloqueios eficientes, relacionando-as aos valores descritos como níveis plasmáticos seguros.

Método - Oitenta e um pacientes de ambos os sexos, submetidos à cirurgia de membros inferiores, receberam aleatoriamente ropivacaína $(n=41)$ ou bupivacaína $(n=40)$ por via peridural lombar associado à anestesia geral. Foram coletadas oito amostras de sangue venoso nos intervalos de tempo: zero (controle), 5, 25, 40, 60, 120, 180 e 240 minutos, e através de cromatografia de gás foram dosadas as concentrações plasmáticas da ropivacaína e da bupivacaína.

Resultados - Não houve diferença estatisticamente significante com relação aos dados antropométricos e variáveis fisiológicas estudadas entre os pacientes que receberam ropivacaína e bupivacaína. As doses médias administradas de ropivacaína e bupivacaína foram 2,35 $\mathrm{mg} \cdot \mathrm{kg}^{-1}$ e 2,13 mg. $\mathrm{kg}^{-1}$, respectivamente, que geraram as concentrações plasmáticas de 2,334 $\mu \mathrm{g} \cdot \mathrm{ml}^{-1}$ e 1,111 $\mu \mathrm{g} \cdot \mathrm{ml}^{-1}$, aos 25 e 40 minutos. Ambas abaixo do nível consideradó seguro $\left(3 \mu \mathrm{g} \cdot \mathrm{kg}^{-1}\right)$.

Conclusões - A administração peridural lombar de ropivacaína e bupivacaína em crianças, nas doses abaixo de $3 \mathrm{mg} \cdot \mathrm{kg}^{-1}$, produz bloqueio anestésico eficaz e determina concentrações plasmáticas que podem ser consideradas seguras.

\footnotetext{
* Recebido do (Received from) Hospital Sarah Brasília, Brasilia, DF

1. Anestesiologista do Hospital Sarah Brasília

2. Bioquímico do Hospital Sarah Brasília

3. Mestre em Estatística do Hospital Sarah Brasilia

4. Coordenador de Anestesiologia da Rede Sarah de Hospitais do ApareIho Locomotor
}

Apresentado (Submitted) em 09 de abril de 2001

Aceito (Accepted) para publicação em 18 de julho de 2001

Correspondencia para (Mail to)

Dra. Verônica Vieira da Costa

Coordenação da Anestesiologia

SMHS Quadra 501 Conjunto: A

70335-901 Brasilia, DF

(C) Sociedade Brasileira de Anestesiologia, 2002
UNITERMOS: ANESTÉSICOS, Local: bupivacaína, ropivacaína; TÉCNICAS ANESTÉSICAS, Regional: peridural

\section{SUMMARY}

Costa VV, Souza DPO, Buzzi M, Mello MC, Saraiva RA - Ropivacaine and Bupivacaine Plasma Concentration During Lumbar Epidural Anesthesia in Children

Background and Objectives - Ropivacaine is the newest local anesthetic drug for clinical practice. Its structure is similar to levogyrous bupivacaine, therefore exibiting low toxicity. Little is known about plasma concentrations that can be reached in children after lumbar epidural administration of these drugs (including racemic bupivacaine). The aim of this study was to evaluate ropivacaine and bupivacaine plasma concentrations following successful lumbar epidural blocks in children, correlating them to known safe plasma levels.

Methods - Eight-one children of both genders, aged between 2 and 16 year, scheduled to undergo lower limbs surgeries, were randomly divided in two groups to receive lumbar epidural ropivacaine $(n=41)$ or bupivacaine $(n=40)$, associated to general anesthesia. Venous blood samples were collected at times 0 (control), 5, 25, 40, 60, 120, 180 and 240 minutes. Ropivacaine and bupivacaine plasma concentrations were measured by gas chromatography.

Results - There was no statistically significant difference in anthropometric and physiological variables between groups. Mean ropivacaine and bupivacaine doses were $2.35 \mathrm{mg} . \mathrm{kg}^{-1}$ and $2.13 \mathrm{mg} \cdot \mathrm{kg}^{-1}$ respectively, leading to peak plasma concentrations of $2.334 \mu \mathrm{g} \cdot \mathrm{ml}^{-1}$ and $1.111 \mu \mathrm{g} \cdot \mathrm{ml}^{-1}$ at 25 and 40 minutes respectively, both below the safety level of $3 \mu \mathrm{g} \cdot \mathrm{ml}^{-1}$.

Conclusions - Lumbar epidural administration of ropivacaine and bupivacaine in children, in doses below $3 \mathrm{mg} \cdot \mathrm{kg}^{-1}$, result in efficient anesthetic blocks and safe plasma concentrations.

KEY WORDS: ANESTHETICS, Local: bupivacaine, ropivacaine; ANESTHETIC TECHNIQUES, Regional, epidural

\section{INTRODUÇÃO}

$A_{\text {ten }}^{\text {ropin }}$ ropivacaína é um anestésico local do tipo amida, que tem sido bastante estudado em adultos e adolescentes ${ }^{1}$. Em crianças, ela tem propriedades que tornam seu uso muito satisfatório, pois produz bloqueio nervoso diferencial com menor bloqueio motor e menor toxicidade cardiovascular e neurológica, o que possibilita a realização de procedimentos ambulatoriais com maior segurança clínica ${ }^{1}$. Recentemente há relatos da eficácia e segurança quando administrado por via caudal ${ }^{2-5}$ e peridural lombar em crianças $^{6,7}$. Durante algum tempo, o único anestésico local disponível para cirurgias ortopédicas de média e longa duração era a bupivacaína. Este agente em sua forma racê- 
mica tem pequeno potencial de toxicidade neurológica e grande potencial de toxicidade cardiológica ${ }^{1,8,9}$.

Aropivacaína foi introduzida para estudos iniciais em 1985, e em 1989, Scott relatou os resultados de seu estudo em que a ropivacaína produziu menos sintomas tóxicos sobre o sistema nervoso central do que a bupivacaína ${ }^{8}$. A farmacocinética da ropivacaína em comparação à da bupivacaína foi investigada após administração venosa e peridural em cães, em que os autores observaram que após injeção venosa, a concentração da ropivacaína declina mais rapidamente durante a fase de eliminação, sugerindo uma maior margem de segurança. Entretanto, após administração peridural, a farmacocinética das duas drogas é semelhante ${ }^{10}$. A ropivacaína, por ter um pequeno efeito vasoconstritor, pode ser administrada sem a adição de adrenalina ${ }^{1}$.

Em humanos adultos, a administração peridural de ropivacaína demonstra absorção bifásica, havendo uma fase inicial rápida com $\left(T_{1 / 2}=14 \mathrm{~min}\right)$ e uma fase subseqüente mais lenta com meia vida terminal em torno de 4,2 horas ${ }^{11,12}$. Com relação às crianças, os parâmetros farmacocinéticos têm sido calculados para diferentes grupos etários submetidos à infusão contínua por via peridural ${ }^{7}$.

O objetivo desse estudo é avaliar as concentrações sangüíneas que podem ser atingidas quando a ropivacaína e a bupivacaína são administradas em bolus, por via peridural lombar em crianças, sendo o bloqueio eficaz, relacionando-se os valores encontrados àqueles descritos como níveis de segurança ${ }^{13,14}$.

\section{MÉTODO}

Após aprovação do Comitê de Ética do Hospital, foram estudados 81 pacientes de ambos os sexos, na faixa etária de 2 a 16 anos, classificados como estado físico ASA I e II, submetidos à cirurgia ortopédica ou plástica reparadora dos membros inferiores sob anestesia geral, associada à peridural lombar. Todos os pacientes receberam midazolam por via oral $\left(0,8 \mathrm{mg} \cdot \mathrm{kg}^{-1}\right) 40$ minutos antes da cirurgia. Aindução dos pacientes de 2 a 8 anos de idade foi realizada com halotano ou sevoflurano, enquanto os acima de 8 anos, foram induzidos com tiopental ( $\left.5 \mathrm{mg} \cdot \mathrm{kg}^{-1}\right)$ ou propofol $\left(3 \mathrm{mg} \cdot \mathrm{kg}^{-1}\right)$. A hidratação per-operatória foi realizada com solução de Ringer com lactato. Após intubação traqueal, a manutenção anestésica foi realizada com anestésico inalatório. Após anestesia geral os pacientes foram posicionados em decúbito lateral para realização da anestesia peridural lombar com agulha Tuohy $50 \times 0,9 \mathrm{~mm}$, sendo administradas aleatoriamente bupivacaína com adrenalina 1:200.000 $(n=40)$ ou ropivacaína $(n=41)$ sem vasoconstritor em concentrações e volumes de acordo com o peso e idade dos pacientes, não excedendo o limite de $3 \mathrm{mg} \cdot \mathrm{kg}^{-1}$.

Excluímos do estudo pacientes portadores de coagulopatias, hepatopatias, cardiopatias e doenças neurológicas.

Foram coletadas 8 amostras de sangue venoso para dosagem do anestésico local, nos seguintes intervalos de tempo: zero (antes da administração do anestésico local), 5, 25, 40, $60,120,180$ e 240 minutos após administração do anestési- co local. Foram feitas análises da concentração plasmática do anestésicolocal por cromatografia a gás e espectrometria de massa (GC-MS), realizadas em um cromatógrafo Hewllett-Packard modelo 6890 interfaceado com detector de massa MSD 6890. Os anestésicos locais bupivacaína e ropivacaína foram extraídos do soro através de uma modificação do método de Tahraoui ${ }^{15}$.

Foram monitorizados continuamente: fração inspirada e final (alveolar) do anestésico inalatório utilizado, freqüência cardíaca, pressão arterial não invasiva, saturação periférica da hemoglobina pelo oxigênio $\left(\mathrm{SpO} \mathrm{O}_{2}\right), \mathrm{CO}_{2}$ expirado $\left(\mathrm{P}_{\mathrm{ET}} \mathrm{CO}_{2}\right)$ e temperatura. Aconfirmação da instalação do bloqueio motor foi realizada através da verificação de dilatação do esfíncter anal, pela diferença de temperatura e vasodilatação entre as regiões anestesiadas e não anestesiadas. A eficácia do bloqueio era confirmada pela concentração alveolar e pelo consumo do anestésico inalatório. Foi considerado eficaz quando a concentração alveolar era sempre menor (submúltiplo) do que a Concentração Alveolar Mínima (CAM).

A avaliação neurológica clínica foi realizada no pré-anestésico, após administração da medicação pré-anestésica e na sala de recuperação pós-anestésica.

As análises estatísticas realizadas foram a análise exploratória dos dados para dose administrada e concentração plasmática encontrada, e a análise de variância, através do teste F de Fisher, para comparação de médias dos dados antropométricos e variáveis fisiológicas estudadas.

\section{RESULTADOS}

Não houve diferença estatística entre os grupos estudados com relação ao peso, idade, sexo e estado físico (Tabelal).

Tabela I - Dados Demográficos

\begin{tabular}{lcc}
\hline Variável & $\begin{array}{c}\text { Bupivacaína } \\
(\mathrm{n}=40)\end{array}$ & $\begin{array}{c}\text { Ropivacaína } \\
(\mathrm{n}=41)\end{array}$ \\
\hline Idade * $^{*}$ & $10,7 \pm 4,05$ & $10,4 \pm 4,13$ \\
Peso * & $34,18 \pm 14,7$ & $37,91 \pm 18,44$ \\
Estado físico ** & \\
ASA I & 22 & 24 \\
ASA II & 18 & 17 \\
Sexo ** & & 20 \\
Masculino & 19 & 21 \\
Feminino & 21 & \\
\hline
\end{tabular}

* Valores expressos pela Média \pm DP

** Resultados expressos pela proporção de casos em cada grupo

Não foi verificada nenhuma falha de bloqueio, o consumo de anestésico inalatório foi pequeno e todos os pacientes foram mantidos com fração expirada final do anestésico inalatório igual ou menor do que 0,5 CAM.

Com relação às variáveis fisiológicas estudadas (pressão arterial, freqüência cardíaca, $\mathrm{ECG}, \mathrm{SpO}_{2}, \mathrm{P}_{\mathrm{ET}} \mathrm{CO}_{2}$ e temperatura), verificou-se que houve estabilidades cardiovascular e 
respiratória nos dois grupos estudados, sem diferenças estatisticamente significantes (Figuras 1,2 e 3). Não houve alteração significativa de temperatura faríngea em nenhum grupo.

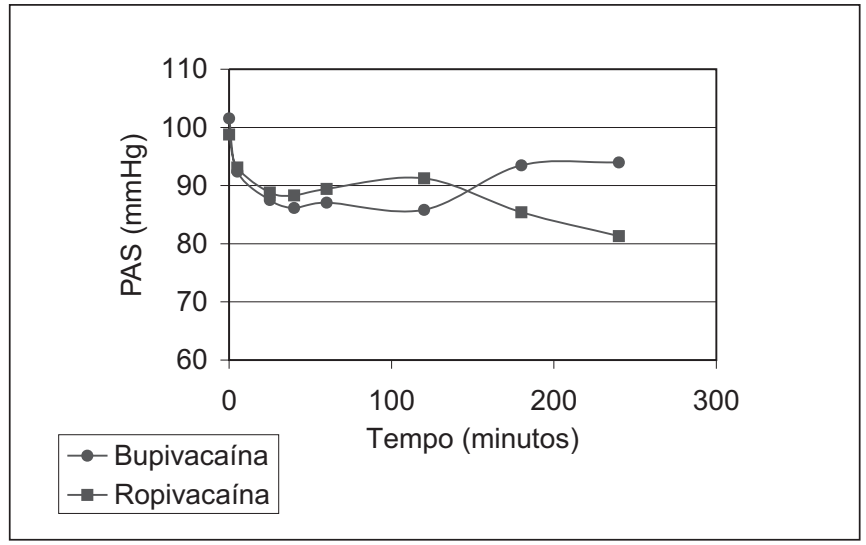

Figura 1 - Pressão Arterial Sistólica nos Dois Grupos Estudados Valor $p=0,593$

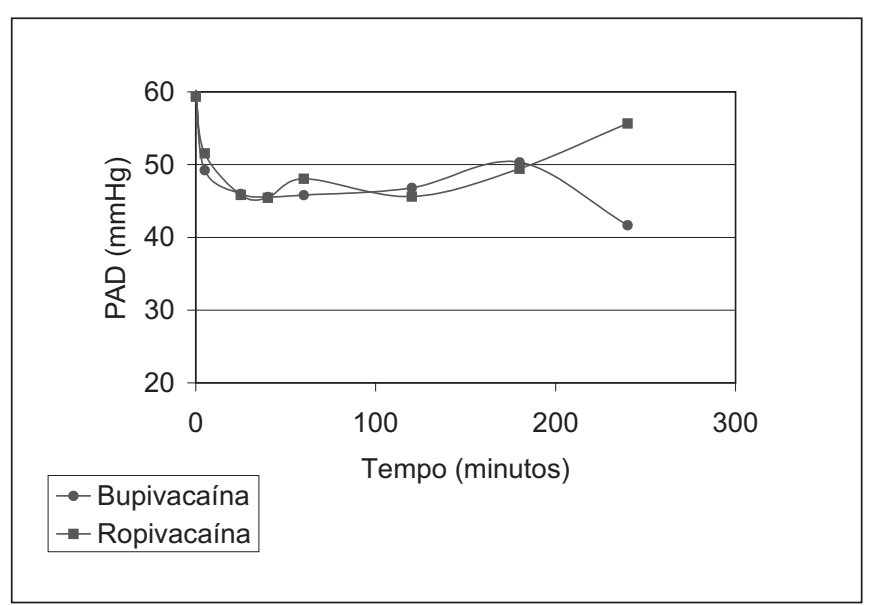

Figura 2 - Pressão Arterial Diastólica nos Dois Grupos Estudados Valor $p=0,486$

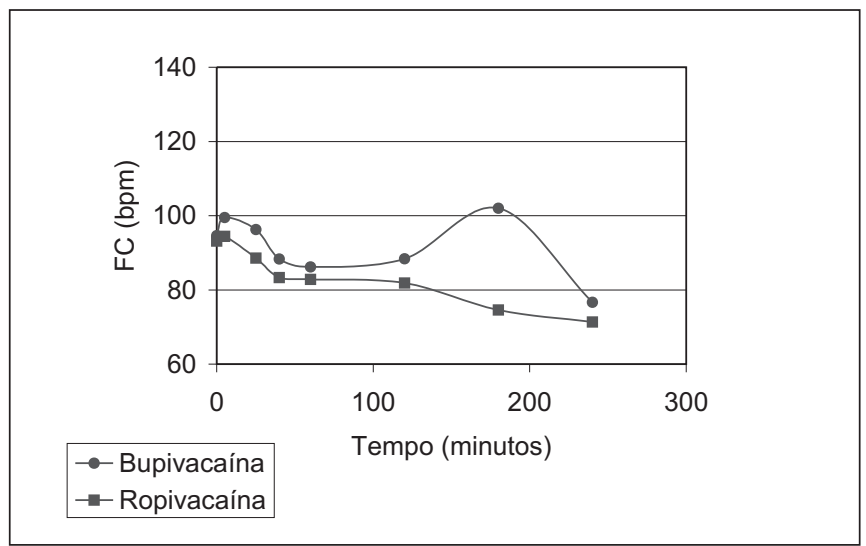

Figura 3 - Freqüência Cardíaca nos Dois Grupos Estudados Valor $p=0,008$

Revista Brasileira de Anestesiologia

Vol. 52, № 1, Janeiro - Fevereiro, 2002
A dose média administrada de ropivacaína foi $2,35 \mathrm{mg} \cdot \mathrm{kg}^{-1}$. Esta dose produziu uma concentração plasmática máxima de $2,334 \mu \mathrm{g} \cdot \mathrm{ml}^{-1}$ aos 40 minutos. Para bupivacaína, a dose média administrada foi $2,13 \mathrm{mg} \cdot \mathrm{kg}^{-1}$, produzindo uma concentração plasmática máxima de $1,111 \mu \mathrm{g} \cdot \mathrm{ml}^{-1}$ aos 25 minutos (Figura 4).

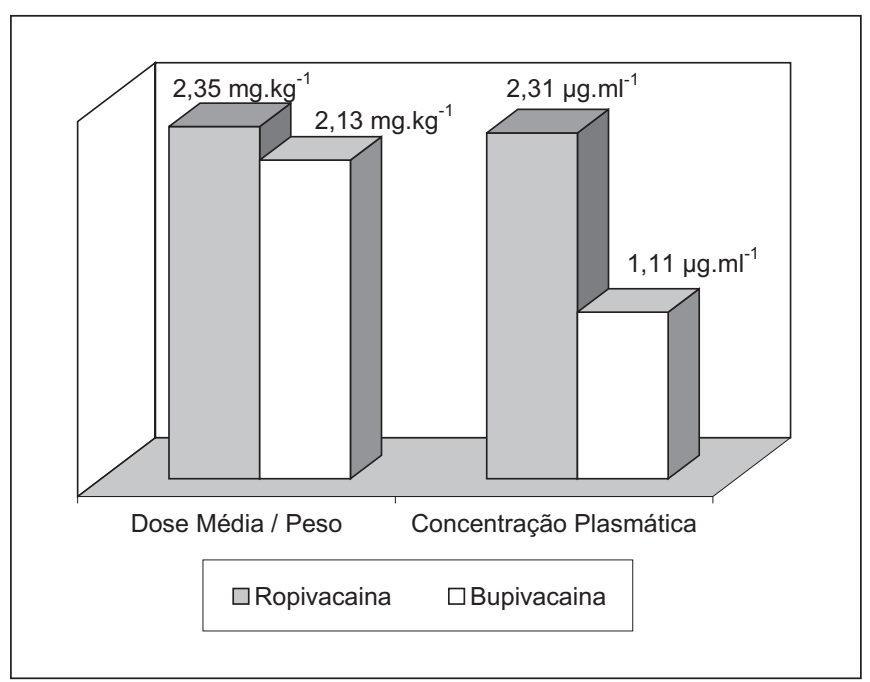

Figura 4 - Relação Dose Média Administrada e Concentração Plasmática Atingida nos Dois Grupos Estudados

A curva da concentração plasmática da ropivacaína mostra que houve um aumento progressivo da concentração até os 40 minutos. A partir daí começa a haver diminuição dos níveis plasmáticos, apresentando baixa concentração plasmática ao término da terceira hora após administração do anestésico local. Com relação à bupivacaína, houve também aumento progressivo até os 25 minutos com diminuição dos níveis a partir desse intervalo de tempo (Figura 5).

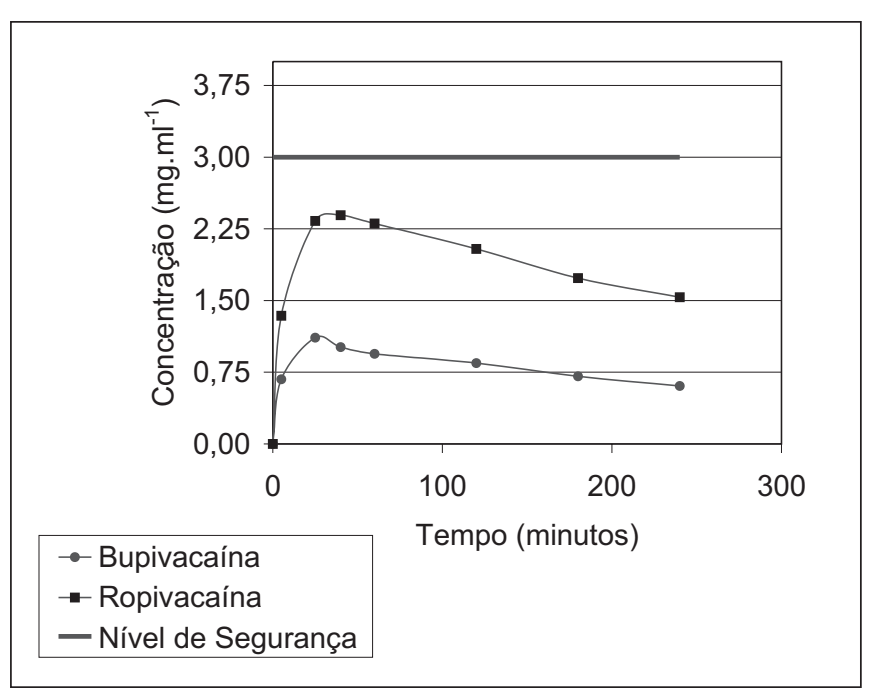

Figura 5 - Concentração Plasmática das Duas Drogas Estudadas 
Na sala de recuperação pós-anestésica, todos os pacientes foram avaliados clinicamente, sem nenhum sinal clínico evidente de alteração neurológica que pudesse indicar reação neurotóxica, ou seja, mantinham o mesmo padrão normal do exame neurológico realizado anteriormente.

\section{DISCUSSÃO}

Os resultados observados confirmam os obtidos por outros autores no que se refere às repercussões sobre as funções cardiovascular e neurológica, segundo os quais as duas drogas foram eficazes e não produziram alteração clínica significativa no sistema cardiovascular e neurológico ${ }^{16}$. No entanto, o estudo foi realizado em pacientes adultos e não foram dosadas as concentrações plasmáticas das duas drogas. Em outro estudo realizado em voluntários adultos ${ }^{8}$, os autores administraram bupivacaína ou ropivacaína por via venosa continuamente até a dose de $150 \mathrm{mg}$ ou até o aparecimento de sinais clínicos ou sintomas de intoxicação. Concluíram que a ropivacaína causa $25 \%$ menos toxicidade do sistema nervoso central e que ambas as drogas aumentam a freqüência cardíaca e a pressão arterial e reduzem o fluxo sangüíneo e a fração de ejeção. Embora tanto a bupivacaína quanto a ropivacaína causem evidência de depressão da condutividade e contratilidade cardíaca, esses sinais aparecem com concentrações menores de bupivacaína do que de ropivacaína ${ }^{8}$. Um estudo anterior realizado em ratos mostrou que ambas as drogas aumentam a freqüência cardíaca e pressão arterial, reduzem o volume sangüíneo e a fração de ejeção ${ }^{17}$. Provavelmente a boa estabilidade cárdiopulmonar observada no nosso estudo seja devido à concentração plasmática estar bem abaixo da encontrada no estudo feito em ratos, que foi $6 \mu \mathrm{g} \cdot \mathrm{ml}^{-1}$ para bupivacaína e $13 \mu \mathrm{g} \cdot \mathrm{ml}^{-1}$ para ropivacaína. O nível plasmático que oferece segurança é de $3 \mu \mathrm{g} \cdot \mathrm{ml}^{-1}$ para ambos os agentes bupivacaína e ropivacaína 13,14. Há relato de aparecimento de sinais e sintomas de toxicidade, em voluntários sadios, após infusão venosa de bupivacaína e ropivacaína, com níveis plasmáticos de ambas as drogas entre 1 e $2 \mu \mathrm{g} \cdot \mathrm{ml}^{-18}$. No entanto, esse mesmo estudo questiona se esses sinais não podem ser atribuídos à ansiedade apresentada pelos voluntários ${ }^{8}$.

Um experimento realizado em cães ${ }^{18}$ mostrou que a dose e concentração plasmática de ropivacaína que poderia levar a convulsão seria de $4,9 \mathrm{mg} \cdot \mathrm{kg}^{-1}$ e $11,4 \mu \mathrm{g} \cdot \mathrm{ml}^{-1}$, respectivamente. Para a bupivacaína a dose encontrada foi de 4,3 $\mathrm{mg} \cdot \mathrm{kg}^{-1}$ e a concentração plasmática de $18 \mu \mathrm{g} \cdot \mathrm{ml}^{-1}$. No nosso estudo as doses empregadas foram bem menores e produziram concentrações plasmáticas bem inferiores àquelas que levaram à convulsão em cães. Com relação ao sistema cardiovascular e pulmonar, tanto a ropivacaína quanto a bupivacaína mostraram boa estabilidade.

A absorção para a circulação após administração peridural foi bifásica, com uma fase inicial rápida, seguida por uma fase mais lenta, o que apóia com estudos anteriores ${ }^{10,13}$. Essa mesma absorção já havia sido descrita anteriormente para a bupivacaína e outros anestésicos locais como lidocaína, e etidocaína em humanos ${ }^{19-21}$ e em macacos ${ }^{22}$.

A concentração plasmática da ropivacaína foi maior do que a da bupivacaína, concordando com alguns estudos realizados anteriormente ${ }^{1,10,12}$. Além da dose administrada de ropivacaína ter sido maior, outra hipótese que pode ser levantada como possível causa seria o fato da ropivacaína ser administrada sem adrenalina, diferentemente da bupivacaína. Em estudos in vitro ${ }^{16}$ foi confirmado que a ropivacaína tem efeito vasoconstritor, mas que esse efeito pode ser mínimo com as doses utilizadas na prática clínica. No nosso estudo, a ropivacaína foi administrada em concentrações e volumes pequenos o que poderia diminuir ainda mais seu efeito vasoconstritor. Outros estudos, no entanto, afirmam que a adição de adrenalina não diminui o pico da concentração plasmática da ropivacaína após injeção peridural ${ }^{1,2}$. O pico da concentração plasmática média da bupivacaína encontrada está de acordo com estudo anterior também realizado em crianças, no qual os autores encontraram um pico que variou de 0,94 a 2,93 $\mu \mathrm{g} \cdot \mathrm{ml}^{-1} 23$. O tempo do pico (25 minutos) também está de acordo com o mesmo estudo, que foi entre $12 \mathrm{e}$ 30 minutos. Após o pico, houve um declínio gradual da concentração, da mesma forma como ocorreu em nosso estudo. A respeito da ropivacaína, não encontramos na literatura nenhum estudo em criança, no qual foi dosada a concentração sangüínea venosa de ropivacaína após administração peridural lombar em bolus. Alguns estudos foram realizados em adultos após infusão venosa ${ }^{8,10,11,24}$, enquanto outros foram realizados em animais ${ }^{17,18}$. A maioria dos estudos realizados em crianças avaliaram a qualidade e altura do bloqueio sensitivo e motor ${ }^{2,3,6}$ e, nesses estudos a via de administração foi a peridural caudal ${ }^{2-6}$ e não a peridural lombar. Na investigação mais recente a respeito a farmacocinética da ropivacaína em crianças ${ }^{7}$, o autor estudou a eficácia clínica e segurança da droga quando administrada via peridural lombar, mas em infusão contínua.

A diferença nos tempos nos quais foram determinados os picos das duas drogas, ou seja, 25 minutos para bupivacaína e 40 minutos para ropivacaína pode ser explicada pela maior solubilidade lipídica da ropivacaína em relação à bupivacaína, conferindo benefício com relação à diminuição da absorção no espaço peridural ${ }^{1}$.

Em conclusão, a administração peridural lombar em bolus de ropivacaína e bupivacaína em crianças, nas doses abaixo de 3 $\mathrm{mg} . \mathrm{kg}^{-1}$, produz bloqueio eficaz e concentrações plasmáticas abaixo das consideradas potencialmente tóxicas, que declinam progressivamente após 40 minutos, permanecendo concentrações sangüíneas mínimas após 4 horas da administração. 


\section{Ropivacaine and Bupivacaine Plasma Concentration During Lumbar Epidural Anesthesia in Children}

Verônica Vieira da Costa, M.D., Denise Pereira de Oliveira Souza, M.D., Marcelo Buzzi, Márcio Corrêa de Mello, Renato Ângelo Saraiva, TSA, M.D.

\section{INTRODUCTION}

Ropivacaine is an amide-type local anesthetic which has been extensively studied in adults and teen agers ${ }^{1}$. It is considered very suitable for children, for inducing a differential nervous block, with less motor block and lower cardiovascular and neurological toxicity, thus allowing safer outpatient procedures ${ }^{1}$. There are recent reports about the efficacy and safety of caudal ${ }^{2-5}$ and lumbar epidural ropivacaine administration in children ${ }^{6,7}$. For some time, bupivacaine was the only local anesthetic available for medium and long duration orthopedic surgeries. In its racemic form, this agent has low neurological and high cardiovascular toxicity potential ${ }^{1,8,9}$

Ropivacaine was introduced for preliminary studies in 1985. In 1989 Scott reported his study's results showing less central nervous system toxicity with this agent compared to bupivacaine ${ }^{8}$. Ropivacaine and bupivacaine had their pharmacokinetics in dogs studied in parallel, when the authors observed that, after intravenous administration, ropivacaine's concentration decreased more rapidly during the elimination phase, suggesting a better safety margin. However, after epidural administration both drugs showed similar pharmacokinetics ${ }^{10}$. Due to its minor vasoconstrictor effect, ropivacaine can be administered without epinephrine ${ }^{1}$. Epidural ropivacaine in adult humans presents a two-phase absorption, with a fast initial phase $\left(T_{1 / 2}=14 \mathrm{~min}\right)$ followed by a slower phase, with terminal half-life of approximately 4.2 hours ${ }^{11,12}$. In children, pharmacokinetic parameters have been calculated for different age groups after epidural continuous infusion ${ }^{7}$.

The purpose of this study was to evaluate plasma concentrations reached with bolus lumbar epidural ropivacaine or bupivacaine in children after successful blocks, correlating them to values described as safe in the literature ${ }^{13,14}$.

\section{METHODS}

After the Hospital's Ethics Committee approval, participated in this study 81 ASA l and II patients of both genders, aged 2 to 16 years, scheduled to undergo lower limb orthopedic or corrective plastic surgeries under general/lumbar epidural anesthesia. All patients were premedicated with oral 0.8 $\mathrm{mg} \cdot \mathrm{kg}^{-1}$ midazolam 40 minutes before surgery. Patients aged 2 to 8 years were induced with halothane or sevoflurane, while those above 8 years of age were induced with venous thiopental $\left(5 \mathrm{mg} \cdot \mathrm{kg}^{-1}\right)$ or propofol $\left(3 \mathrm{mg} \cdot \mathrm{kg}^{-1}\right)$. Perioperative hydration was made with lactated Ringer's solution. After tracheal intubation, anesthesia was maintained with inhalational anesthetics. After general anesthesia, patients were placed in the lateral position for lumbar epidural puncture with a $50 \times 0.9 \mathrm{~mm}$ Tuohy needle. Bupivacaine with epinephrine $1: 200.000(n=40)$ or plain ropivacaine $(n=41)$ were randomly administered in volumes and concentrations according to patients age and weight, not exceeding $3 \mathrm{mg} \cdot \mathrm{kg}^{-1}$. Exclusion criteria were coagulopathies, hepatopathies, cardiopathies and neurological diseases.

Venous blood samples were collected for local anesthetics measurement in the following moments: zero (before local anesthetic injection), 5, 25, 40, 50, 60, 120, 180 and 240 minutes after local anesthetics administration. Local anesthetics plasma concentrations were measured by gas chromatography and mass spectometry (GC-MS), performed in a Hewlett-Packard model 6890 chromatographer interfaced with a mass detector MSD 6890. Bupivacaine and ropivacaine were extracted from the serum through a modified Tahraoui method ${ }^{15}$.

The following parameters were continuously monitored: inhalational anesthetics inspired and end tidal (alveolar) fractions, heart rate, non invasive blood pressure, oxygen peripheral saturation $\left(\mathrm{SpO}_{2}\right)$, expired $\mathrm{CO}_{2}\left(\mathrm{P}_{\mathrm{ET}} \mathrm{CO}_{2}\right)$ and temperature. Blockade onset was confirmed by anal sphincter relaxation, as well as temperature and vasodilatation differences between anesthetized and non anesthetized areas. Blockade success was indirectly confirmed by inhalational anesthetics alveolar concentration (when kept always below minimum alveolar concentration - MAC) and consumption. Clinical neurological evaluations were performed in the preanesthetic visit, after premedication and in the post-anesthetic recovery unit.

Statistical analysis were data exploratory analysis for administered doses and plasma concentrations of local anesthetics, as well as analysis of variance through Fisher's $F$ test to compare mean demographics and physiological variables.

\section{RESULTS}

There was no statistical difference between groups in weight, age, gender and physical status (Table I).

Table I - Demographics Data

\begin{tabular}{lcc}
\hline Variables & $\begin{array}{c}\text { Bupivacaine } \\
(\mathrm{n}=40)\end{array}$ & $\begin{array}{c}\text { Ropivacaine } \\
(\mathrm{n}=41)\end{array}$ \\
\hline Age $^{*}$ & $10.7 \pm 4.05$ & $10.4 \pm 4.13$ \\
Weight $^{*}$ & $34.18 \pm 14.7$ & $37.91 \pm 18.44$ \\
Physical status ** & & \\
$\quad$ ASA I & 22 & 24 \\
ASA II & 18 & 17 \\
Gender ** & & \\
$\quad$ Male & 19 & 20 \\
Female & 21 & 21 \\
\hline
\end{tabular}

* Values expressed in Mean \pm SD

${ }^{* *}$ Results expressed in percentages for each group 
There was no blockade failure. Inhalational anesthetics consumption was low and all patients were maintained with end expired fractions equal to or lower than 0.5 MAC.

Physiological variables (blood pressure, heart rate, ECG, $\mathrm{SpO}_{2}, \mathrm{P}_{\mathrm{ET}} \mathrm{CO}_{2}$ and temperature) showed good cardiovascular and respiratory stability in both groups with no statistically significant difference (Figures 1, 2 and 3). There was no significant change in pharyngeal temperature in both groups. Mean ropivacaine dose was $2.35 \mathrm{mg} \cdot \mathrm{kg}^{-1}$, which produced a maximum plasma concentration of $2.334 \mu \mathrm{g} \cdot \mathrm{ml}^{-1}$ at $40 \mathrm{~min}-$ utes. Mean bupivacaine dose was $2.13 \mathrm{mg}^{\mathrm{kg}}{ }^{-1}$, which produced a maximum plasma concentration of $1.111 \mu \mathrm{g} \cdot \mathrm{ml}^{-1}$ at 25 minutes (Figure 4).

Ropivacaine plasma concentration curve shows that there has been a progressive concentration increase for $40 \mathrm{~min}$ utes. Then, plasma levels started to decrease and showed a low plasma concentration at the end of the third hour after local anesthetics administration. Bupivacaine levels has shown a progressive increase for 25 minutes followed by a decrease as well (Figure 5).

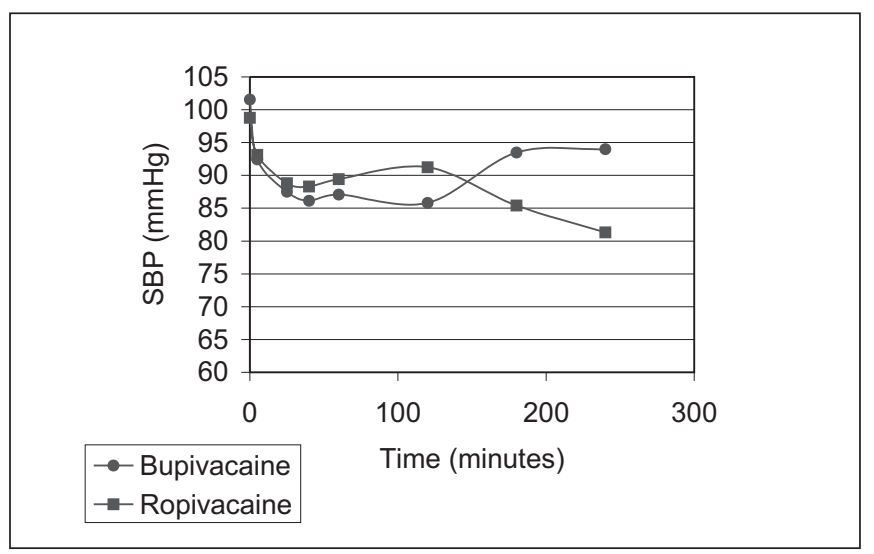

Figure 1 - Systolic Blood Pressure for Both Groups $P$ value $=0.593$

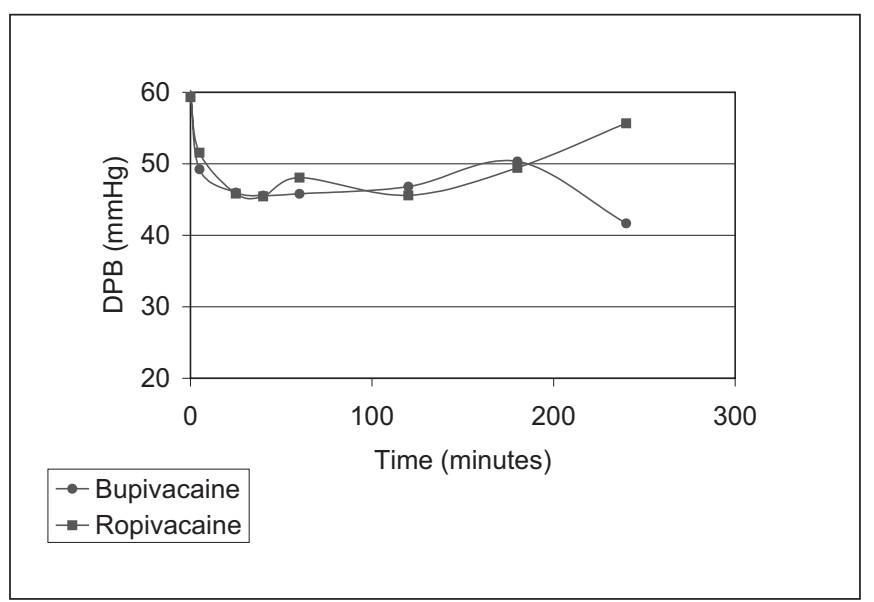

Figure 2 - Diastolic Blood Pressure for Both Groups $P$ value $=0.486$

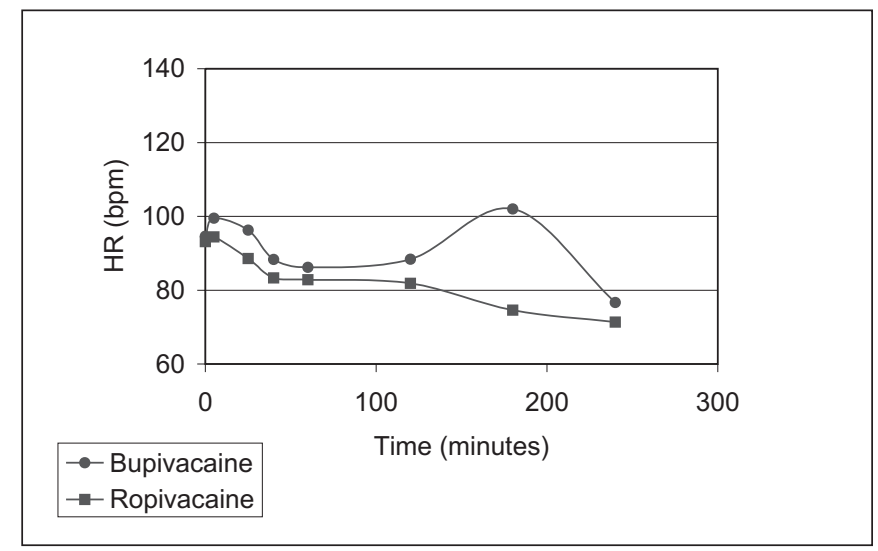

Figure 3 - Heart Rate for Both groups

$P$ value -0.08

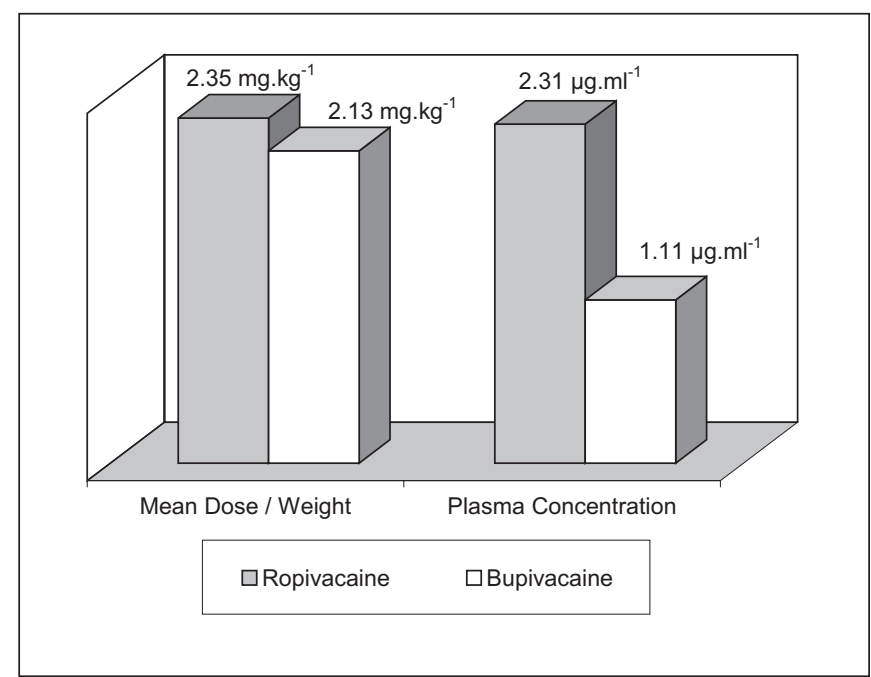

Figure 4 - Mean Dose and Plasma Concentration Ratio for Both Groups

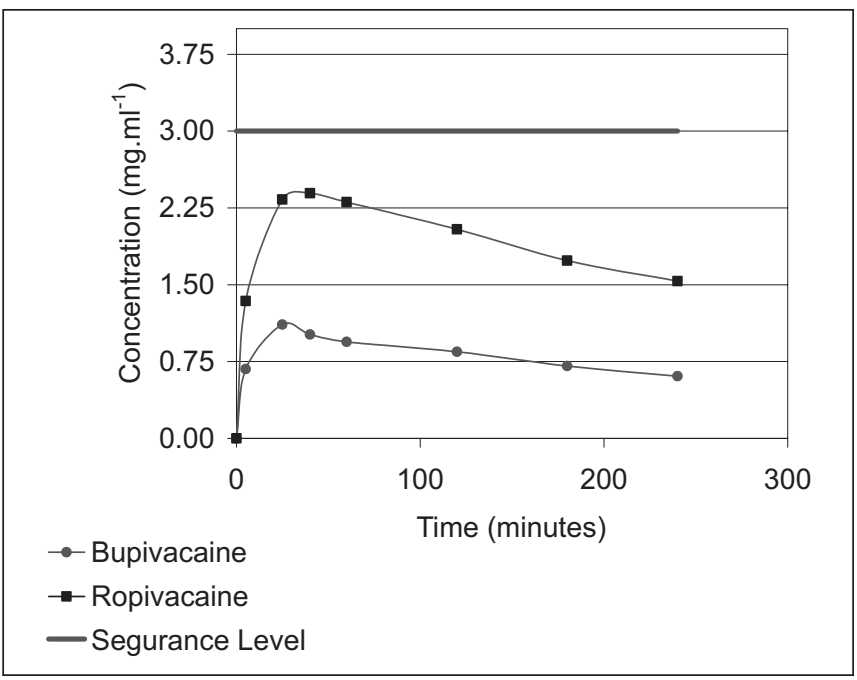

Figure 5 - Plasma Concentration of Both Drugs Vol. 52, № 1, Janeiro - Fevereiro, 2002 
All patients were clinically evaluated in PACU, without signs of neurological changes that could indicate neurotoxic reaction, that is, maintaining the same normal pattern of the initial neurological evaluation.

\section{DISCUSSION}

Our results confirm those obtained by other authors in which both drugs produced effective blockades without significant cardiovascular and neurological changes ${ }^{16}$. This study, however, was performed in adult patients and drug plasma concentrations were not measured. In a different study with adult volunteers ${ }^{8}$, intravenous bupivacaine or ropivacaine were continuously administered until $150 \mathrm{mg}$ or the appearance of clinical intoxication signs or symptoms. The conclusion was that ropivacaine causes $25 \%$ less central nervous system toxicity and that both drugs increase heart rate and blood pressure, decreasing blood flow and ejection fraction. Although bupivacaine and ropivacaine cause cardiac conductivity and contractility depression, such signs appear in lower bupivacaine concentrations as compared to ropivacaine ${ }^{8}$. A previous study in rats has shown that both drugs increase heart rate and blood pressure, decreasing blood volume and ejection fraction ${ }^{17}$. It is possible that the good cardiopulmonary stability observed in our study has been due to plasma concentrations for below that found in the study with rats, which was $6 \mu \mathrm{g} \cdot \mathrm{ml}^{-1}$ for bupivacaine and 13 $\mu \mathrm{g} \cdot \mathrm{ml}^{-1}$ for ropivacaine. Safe plasma level is $3 \mu \mathrm{g} \cdot \mathrm{ml}^{-1}$ for both agents ${ }^{13,14}$. There is a report on toxicity signs and symptoms in healthy volunteers after bupivacaine and ropivacaine intravenous infusion with plasma levels between 1 and 2 $\mu \mathrm{g} \cdot \mathrm{ml}^{-1} 8$. The same study, however, argues whether such signs could be attributed to the anxiety shown by those volunteers ${ }^{8}$.

An experiment with dogs ${ }^{18}$ showed that ropivacaine dose and plasma concentration leading to seizure would be 4.9 $\mathrm{mg} . \mathrm{kg}^{-1}$ and $11.4 \mu \mathrm{g} . \mathrm{kg}^{-1}$, respectively. For bupivacaine this dose would be $4.3 \mathrm{mg} \cdot \mathrm{kg}^{-1}$ and plasma concentration 18 $\mu \mathrm{g} \cdot \mathrm{ml}^{-1}$. In our study, the doses used were lower, producing plasma concentrations for below those leading to seizure in dogs. Ropivacaine and bupivacaine showed good cardiovascular and respiratory stability. Absorption to circulation after epidural administration followed a two-phase pattern, with an initial rapid phase followed by a slower one accordance with previous studies ${ }^{10,13}$. This same kind of absorption had been previously described for bupivacaine and other local anesthetics, such as lidocaine and ethiocaine, in humans $^{19-21}$ and monkeys ${ }^{22}$.

Ropivacaine reached higher plasma concentrations than bupivacaine, in agreement with some previous studies ${ }^{1,10,12}$. In addition to a higher ropivacaine dose, other possible reason could be that, as opposed to bupivacaine, ropivacaine was administered without epinephrine. In vitro studies ${ }^{16}$ confirmed that ropivacaine has a vasoconstrictor effect, but this effect might be negligible with the concentration used in clinical practice. In our study, ropivacaine was administered in low volumes and concentrations which could further de-

Revista Brasileira de Anestesiologia

Vol. 52, No 1, Janeiro - Fevereiro, 2002 crease its vasoconstrictor effect. Other studies, however, state that the addiction of epinephrine will not decrease ropivacaine's plasma peak concentration after epidural injection ${ }^{1,2}$. Mean bupivacaine plasma concentration peak found here is in line with a previous study also performed in children where the authors found a peak varying from 0.94 to $2.93 \mu \mathrm{g} \cdot \mathrm{ml}^{-123}$. Peak time (25 minutes) is also in agreement with the same study, where it ranged between 12 and 30 minutes. After that, there has been a gradual concentration decrease, similarly to what was observed in our study. We have found no study in the literature performed with ropivacaine in children measuring its venous blood concentration after bolus lumbar epidural administration. Some studies were performed in adults after intravenous infusion ${ }^{8,10,11,24}$, while others were performed in animals 17,18. Most studies in children have evaluated sensory and motor block quality and level ${ }^{2,3,6}$ with caudal administration route ${ }^{2-6}$, and not lumbar epidural. In a more recent investigation of ropivacaine pharmacokinetics in children ${ }^{7}$, the author studied clinical efficacy and safety of lumbar epidural administration, but in continuous infusion.

Difference in time elapsed for both drugs peak, that is, $25 \mathrm{~min}$ utes for bupivacaine and 40 minutes for ropivacaine, may be explained by a better ropivacaine lipidic solubility as compared to bupivacaine, which is beneficial for decreasing epidural space absorption ${ }^{1}$.

In conclusion, bolus ropivacaine or bupivacaine lumbar epidural administration in children, in doses below $3 \mathrm{mg} \cdot \mathrm{kg}^{-1}$, produces effective blockades and plasma concentrations below those considered potentially toxic, which progressively decrease after 40 minutes and remain in negligible levels after 4 hours of administration.

\section{REFERÊNCIAS - REFERENCES}

01. McClure JH - Ropivacaine. Br J Anaesth, 1996;76:300-307.

02. Conceição MJ, Coelho L - Caudal anaesthesia with $0.375 \%$ ropivacaine or $0.375 \%$ bupivacaine in paediatric patients. $\mathrm{Br} \mathrm{J}$ Anaesth, 1998;80:507-508.

03. Conceição MJ, Coelho L, Khalil M - Ropivacaine $0.25 \%$ compared with bupivacaine $0.25 \%$ by the caudal route. Paediatr Anaesth, 1999;92:229-233

04. Habre W, Bergesio R, Johnson C et al - Plasma ropivacaine concentrations following caudal analgesia in children. Anesthesiology, 1998;89:AI245.

05. Habre W, Bergesio R, Johnson C et al - Pharmacokinetics of ropivacaine following caudal analgesia in children. Paediatr Anaesth, 2000;10:143-147.

06. Ivani G, Lampugnani E, De Negri $P$ et al - Ropivacaine vs bupivacaine in major surgery in infants. Can J Anaesth, 1999; 46:467-469.

07. Hansen TG, llett KF, Lim SI, Reid C et al - Pharmacokinetics and clinical efficacy of long-term epidural ropivacaine infusion in children. Br J Anaesth, 2000;85:347-353.

08. Scott DB, Lee A, Fagan D et al - Acute toxicity of ropivacaine compared with trat of bupivacaine. Anesth Analg, 1989;69: 563-569. 
09. Albright GA - Cardiac arrest following regional anesthesia with etidocaine and bupivacaine. Anesthesiology, 1979;51:285-287.

10. Arthur GR, Feldman HS, Covino BG - Comparative pharmacokinetics of bupivacaine and ropivacaine, a new amide local anesthetic. Anesth Analg, 1988;67:1053-1058.

11. Katz JA, Bridenbaugh PO, Knarr DC et al - Pharmacodynamics and pharmacokinetics of peridural ropivacaine in humans. Anesth Analg, 1990;70:16-21.

12. Morrison LMM, Emanuelsson BM, McClure JH et al - Efficacy and kinetics of extradural ropivacaine comparison with bupivacaine. Br J Anaesth, 1994;72:164-169.

13. Emanuelsson BMK, Person J, Alm C et al - Systemic absorption and block after epidural injection of ropivacaine in healthy volunteers. Anesthesiology, 1997;87:1309-1317.

14. Frawley G, Ragg P - Plasma concentrations of bupivacaine after combined spinal epidural anaesthesia in infants and neonates. Paediatr Anaesth, 2000;10:619-625.

15. Tahraoui A, Watson DG, Skellem GG et al - Comparative study of the determination of bupivacaine in human plasma by gas chromatography - mass spectrometry and high performance liquid chromatography. J Pharm Biomed Anal, 1996;15:251-257.

16. Nakamura K, Toda $H$, Kakuyama $M$ et al - Direct vascular effect of ropivacaine in femoral artery and vein of the dog. Acta Anaesthesiol Scand, 1993;37:269-273.

17. Pitkanen M, Covino BG, Feldman HS et al - Chronotropic and inotropic effects of ropivacaine, bupivacaine, and lidocaine in the spontaneously beating and electrically paced isolated, perfused rabbit heart. Reg Anesth, 1992;17:183-192.

18. Feldman HS, Arthur GR, Covino BG - Comparative systemic toxicity of convulsant and supraconvulsant doses of intravenous ropivacaine, bupivacaine lidocaine in the conscious dog. Anesth Analg, 1989;69:794-801.

19. Tucher GT, Mather LE - Pharmacokinetics of local anaesthetic agents. Br J Anaesth, 1975;47:213-214.

20. Burm AGL, Vermeulen NPE, Van Kleef JW et al - Pharmacokinetics of lignocaine and bupivacaine in surgical patients following epidural administration. Simultaneous investigations of absorption and disposition kinetics using stable isotopes. Clin Pharmacokinet, 1979;13:191-203.

21. Tucker GT, Mather LE - Clinical pharmacokinetics of local anaesthetics. Clin Pharmacokinet, 1979;4:241-278.

22. Katz JÁ, Sehlhorst CS, Thompson GA et al - Pharmacokinetics of intravenous and epidural ropivacaine in the rhesus monkey. Biopharm Drug Disp, 1993;14:579-588.

23. Eyres RL, Hastings C, Brown TCK - Plasma bupivacaine concentrations following lumbar epidural anaesthesia in children. Anaesth Intensive Care, 1986;14:131-134.

24. Lee $A$, Fagan D, Lamont $M$ et al- Disposition kinetics of ropivacaine in humans. Anesth Analg, 1989;69:736-738.

\section{RESUMEN}

Costa VV, Souza DPO, Buzzi M, Mello MC, Saraiva RA - Concentración Plasmática de Ropivacaína Durante Anestesia Peridural Lumbar en Niños

Justificativa y Objetivos - La ropivacaína es el más nuevo anestésico local de uso en la práctica clínica. Su estructura es semejante a la forma levógira de la bupivacaína, teniendo por tanto baja toxicidad. Los valores de las concentraciones plasmáticas que pueden ser alcanzados en niños, con el uso de esta droga y también de la bupivacaína (mismo la forma racemica) administradas por vía peridural lumbar, son aun poco conocidos. O objetivo de ese estudio fue evaluar las concentraciones sanguíneas de ropivacaína y bupivacaína por vía peridural lumbar en niños, en bloqueos eficientes, relacionándolas a los valores descritos como niveles plasmáticos seguros.

Método - Ochenta y un pacientes de ambos sexos, sometidos a cirugía de miembros inferiores, recibieron aleatoriamente ropivacaína $(n=41)$ o bupivacaína $(n=40)$ por vía peridural lumbar asociado a anestesia general. Fueron colectadas ocho muestras de sangre venoso en los intervalos de tiempo: cero (control), 5, 25, 40, 60, 120, 180 y 240 minutos, y a través de cromatografia de gas fueron dosificadas las concentraciones plasmáticas de la ropivacaína y de la bupivacaína.

Resultados - No hubo diferencia estadísticamente significante con relación a los datos antropométricos y variables fisiológicas estudiadas entre los pacientes que recibieron ropivacaína y bupivacaína. Las dosis medias administradas de ropivacaína y bupivacaína fueron $2,35 \mathrm{mg} \cdot \mathrm{kg}^{-1}$ e 2,13 $\mathrm{mg} \cdot \mathrm{kg}^{-1}$, respectivamente, que generaron las concentraciones

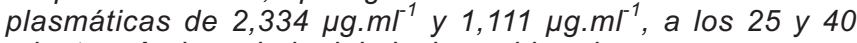
minutos. Ambas abajo del nivel considerado seguro.

Conclusiones - La administración peridural lumbar de ropivacaína y bupivacaína en niños, en las dosis abajo de 3 $\mathrm{mg} \cdot \mathrm{kg}^{-1}$, produce bloqueo anestésico eficaz y determina concentraciones plasmáticas que pueden ser consideradas seguras. 